\title{
ANALYSIS OF THE APPLICATION OF LASER RADIATION IN THE PROCESS OF ORTHODONTIC TOOTH MOVEMENT AND SUGGESTIONS ABOUT THE IMPROVEMENT OF TECHNOLOGY
}

Olena Savchenko

savchenko@mail.ua

Summary. The process of comprehensive orthodontic treatment is characterized by a long-term duration and high probability of the development of adverse effects. Researchers have proven that lasers have a positive effect in the process of orthodontic tooth movement, but the issue of selecting appropriate laser radiation characteristics and developing new modified approaches to the application of laser radiation requires further research.

The aim of the study is to analyze the results of the study on laser radiation application in the process of orthodontic tooth movement, and to make recommendations for the design of a new model of low-level laser device.

Materials and Methods. Content analysis of scientific publications devoted to the investigated problem has allowed us to highlight the works in which solution to this problem is covered to the fullest extent possible and has given the author an opportunity to make their own recommendations for the improvement of laser radiation systems and the development of an analog which would be more mobile in terms of construction and more clinically effective.

Results of the study and their discussion. Most researchers confirm the effectiveness of laser radiation in the process of orthodontic tooth movement, although some of them did not notice any positive effect. According to the first studies, laser radiation favors the fusion of mononuclear macrophages for the maturation of osteoblastic cells, an increase in the quantity of differentiated osteoblastic cells and bone formation centers, and the acceleration of mineralized bone tissue formation in the stretching area. In other studies, the following was noted: an increase of the collagen synthesis, a positive effect on the metalloproteinase-9, cathepsin K and alpha (v) beta (3) integrin expression, and maintenance of initial parameters of bone mineral density. Further studies largely confirmed the preliminary results of tooth movement rate increase by $20-40 \%$ and showed the acceleration of pulp tissues regeneration, thus minimizing the risk of irreversible complications and a significant reduction of pain sensations. Following the results of the conducted analyses and on the basis of own practical experience, the author emphasizes the following recommendations for the development of a new model of low-level laser device: 1) using luminophores in the laser construction and selecting handpieces with different output parameters; 2) using 
the technology of selective dimensional metal plates' etching; 3) constructing knockdown laser systems: 4) the wavelength must be $810 \mathrm{~nm}$.

Conclusions. In order to determine the mechanism of the effect laser radiation has on the orthodontic tooth movement, it is necessary to carry out additional morphometric, X-ray, histological, and clinical studies.

Keywords: laser radiation; tooth movement; macrophage: osteoblast; bone formation.

\section{INTRODUCTION}

In most cases comprehensive orthodontic treatment of patients is a rather long process, duration of which depends on a number of determinants: number of stages in the treatment algorithm, complexity of orthodontic pathology, patient's age, initial characteristics of the dento-alveolar system, oral tissue response to a particular type of iatrogenic intervention $[1,3,7,9)$. In addition, in the course of the use of orthodontic equipment, specific conditions are created for the development of possible adverse effects of treatment, such as gingivitis, carious lesions; as it is hard to carry out adequate hygienic control, there is root resorption, excessive reduction of the level of jaw bone tissue due to remodeling $[10,13,14]$. Finally, in order to avoid the risk of undesirable effects of iatrogenic interventions and to accelerate the process of controlled orthodontic therapy in conjunction with orthodontic procedures, a number of auxiliary exposure methods have been developed which allow one to achieve a more predictable clinical outcome, contribute to minimizing inflammatory processes, and in general allow one not only to systematically approach the process of dental rehabilitation, but also make it more acceptable and less uncomfortable for patients. The potential or summational positive effect of treatment is achieved when carrying out the orthodontic phase and its adjacent approaches, such as orthodontically driven corticotomy,local administration of prostaglandins and osteocalcin, the use of ultrasound, laser radiation, electromagnetic field, or electric current.

Among the first scientists who studied positive effects of lasers on the process of orthodontic tooth movement were Kawasaki and Shimizu, who explained this by accelerative remodeling of the alveolar bone, which is manifested in the increase of the number of osteoblasts, increased levels of cell proliferation in the periodontal ligament space, and mineralized bone tissue formation [8]. In addition, the authors managed to also prove another, no less significant fact: the use of laser radiation with the control of its initial parameters and compliance with a particular biological range does not cause negative changes directly in the area of laser 
exposure and in the bodies of studied animals in general. However, the issue of selecting appropriate characteristics of laser radiation and the development of new modified approaches in order to improve modern technologies in dental practice remains a topical scientific and practical aspect which requires further research in this industry.

The aim of the study was to analyze the effectiveness of using lasers in the process of orthodontic tooth movement and to highlight perspective aspects of the improvement of existing low-level laser radiation systems for the development of an analog which would be more mobile in terms of construction and more clinically effective.

\section{MATERIALS AND METHODS}

Scientific publications devoted to the issue of expediency and effectiveness of the use of laser technologies in the dental - and in particular in orthodontic - practice for the purpose of optimizing the process of orthodontic tooth movement were analyzed in the course of the study. We searched for material using keywords in the Google Scholar search system. In the course of content analysis, we selected those research papers that most fully covered aspects related to the main aim of the study, that is, the issue of laser output parameters for orthodontic movement, clinical signs, and biological markers that confirm the effectiveness of the use of this technology in particular clinical conditions, as well as the prospects of improving existing low-level laser radiation systems. Registration of laser output parameters in a number of studies allowed us to determine those parameters which varied most of all and therefore could lead to the achievement of a particular clinical outcome. On the basis of the conducted analysis, we made a number of own recommendations, which can serve as a basis for the future improvement of laser radiation systems and the development of an analog which would be more mobile in terms of construction and more clinically effective. Numerical parameters obtained in the course of the analysis of previous publications were categorized and grouped in tabular form using Microsoft Excel 2016 (Microsoft Office 2016).

\section{RESULTS OF THE STUDIES AND THEIR DISCUSSION}

The dynamics of orthodontic tooth movement depends on the rate of bone remodeling $[1,4,5,8,10]$. This process does not start immediately on the first day 
of applying mechanical load to a tooth, but it is, in turn, connected with the phases of activation of osteoclastic and osteoblastic cells. That is why, according to Kawasaki and Shimizu, it is in the early period of orthodontic intervention that laser radiation contributes to the process of tooth movement most of all. Such an effect, according to the authors, is observed because the effect of a laser contributes to the fusion of mononuclear macrophages for the maturation of osteoclastic cells [8]. A similar effect is observed in case of local administration of vitamin 1,25-(OH)2D3, which also results in a significant increase in the number of multinucleated bone marrow cells. Among other things, Kawasaki and Shimizu found that not only does laser radiation contribute to faster osteoclast activation, but also increases the rate of mineralized bone tissue formation in the stretching area 1.7 times. This effect is caused by the effects of radiation on the increase in the number of more differentiated osteoblastic cells and formation of bone formation centers. It must be noted that in many studies that were conducted earlier on the effects of lasers on processes associated with orthodontic tooth movement, the protocol was similar to the one primarily described by Kawasaki and Shimizu, which involves contact application of fiber optic to soft tissues directly around the teeth that are subject to disposition $[2,5,10,12]$. Separate studies on the use of low-level laser radiation indicate that not only does this method contribute to the acceleration of bone tissue regeneration process, but also to the increase of collagen synthesis, which, in turn, is the main matrix protein in the bone structure. In particular, this effect was registered when using lasers in the conditions of palatal suture expansion. S. Fujita (2008), in turn, attributed this effect of low-level laser radiation on the rate of orthodontic movement to RANK and RANKL induction, which was confirmed by the corresponding positive results of immune responses [5]. M. Yamaguchi enhanced the results of S. Fujita's study (2008), having found that low-level lasers also have a positive effect on the metalloproteinase-9, cathepsin K, and alpha (v) beta (3) integrin expression. All of the above factors are necessary for the process of osteoclastogenesis [13]. In the course of studies conducted by Japanese scientists, not only did they manage to confirm stimulation of expression of the corresponding bone markers with the help of a laser, but also its positive effect on the preservation of the initial parameters of bone mineral density during orthodontic movement. When using a laser with the following output parameters: $780 \mathrm{~nm}, 20 \mathrm{mV}, 10 \mathrm{~s}, 5 \mathrm{~J} / \mathrm{cm} 2$, M. V., da Silva Sousa and colleagues (2012) found a statistically significant difference in the dynamics of movement of canines over 4 months of observation [3]. At the same time, various bone and root resorptions in teeth that were subject to mechanical loads were almost the same both in cases where a laser was used, and in cases where it was not used.

However, as a result of a double-blind, randomized, placebo-controlled, casecontrol clinical trial conducted by W. Limpanichkul and colleagues (2006), the 
scientists failed to prove the effect of low-level laser therapy on the process of orthodontic tooth movement, but at the same time, they noted that energy density of $25 \mathrm{~J} / \mathrm{cm} 3$, which was used in the experiment, was apparently too small to provoke the occurrence of both an inhibition effect or the expected stimulatory effect [10]. In order to control the process of orthodontic movement of canines, the researchers used a GaAlAs laser with a wavelength of $860 \mathrm{~nm}$ and the following output parameters: $100 \mathrm{mV}$, a spectral area of $0.09 \mathrm{~cm} 2$, a power of $1.11 \mathrm{~V} / \mathrm{cm} 2$, a dose of $2.3 \mathrm{~J} /$ point, and energy density of $25 \mathrm{~J} / \mathrm{cm} 2 /$ section. M. Marquezan (2010) also noted that there is no significant clinical effectiveness when using a laser in the process of orthodontic tooth movement. In the course of the use of two treatment protocols (in case of daily exposure and only at early stages), the authors noted that there was a progressive increase in the number of osteoclasts and immature collagen at an early stage of orthodontic treatment, however this effect was leveled out in the follow-up period due to the fact that the reparation process in the area of tooth strain slowed down [11].

Unlike W. Limpanichkul and colleagues, Cruz and co-authors (2004) confirmed the effect of low-level laser radiation on the process of controlled orthodontic tooth movement, which manifested itself in the acceleration of such a disposition, and thus contributed to a reduction of treatment duration [2]. At the same time, the researchers were using a device with somewhat lower output parameters, but according to a modified algorithm of the procedure. Genc and colleagues (2012) received significantly higher results of orthodontic tooth movement in a research group where low-level laser radiation was used on the $7 ; 14 ; 21 ; 28$, and 35 day of monitoring compared with the control group. Thus, they managed to confirm the preliminary results obtained during the studies conducted by Saito and Shimizu, Youssel and colleagues, Kawasaki and Shimizu, in which the average range of increase in the rate of tooth movement during orthodontic treatment amounted to 20-40\%. It is also important to note that Genc and colleagues (2012) also pointed out the results of previous studies conducted by W. Limpanichkul, which indicated that laser radiation has no effect on orthodontic movement. The authors interpreted such a difference in the effect of laser radiation as the dependence of the desired clinical effect on a radiation dose and the duration of the effect of a physical factor [7]. Among other things, Genc also noted the importance of registering the level of nitrogen oxide as a marker of the response of periodontal tissues to the effect of orthodontic forces. In the author's study, the researchers did not find any statistical difference between the nitrite levels in the study group and control group. Even in the context of the already proven fact that the use of laser radiation contributes to the reparation of bone tissue in the area of exposure, the aspect of the required radiation dose is still unresolved, and it is still unknown which of them has the greatest effect on the corresponding cell lines, thereby stimulating healing effect. At the same time, it is also important to carry out studies in order to check whether 
initial settings of the laser can cause damage to the surrounding tissues and the development of toxic effects.

L. Abi-Ramia (2012) confirmed the fact that orthodontic tooth movement causes the appearance of reactive pulp hyperemia as a result of the effect of mechanical forces, but the use of low-level laser radiation contributes to the process of faster reparation of pulp tissues, and thus minimizes the risk of irreversible complications [1]. In the group where the effect of mechanical disposition without additional use of lasers was studied, it was found that an odontoblast layer inside teeth was more disorganized; at the same time, the researchers observed undifferentiated cells and capillaries overfilled with blood. G. Doshi-Mehta and W. A. Bhad-Patil (2013) were able not only to confirm the increase in the dynamics of tooth movement by an average of $30 \%$ (29\% on the upper jaw and $31 \%$ on the lower jaw), but also a significant reduction of pain sensations according to visual analogue scales on the third day of using an orthodontic appliance [4]. At the same time, in comparison with the 3rd and 30th days of observation, pain score in the experimental group where laser was not used decreased from 2.15 to 1.5 , whereas in the control group it changed from 0.25 to 0.5 . As a result of systematic review and meta-analysis conducted by M. K. Ge and colleagues (2014), in which they managed to analyze 173 studies with a total of 211 participants, it was found that in case of additional use of lasers, acceleration of orthodontic tooth movement was observed in the period of 7 days and 2 months; at the same time, the authors noted higher clinical effectiveness of lower energy density compared with those exceeding $20 \mathrm{~J} / \mathrm{cm} 2$ [6].

An analysis of literary publications devoted to the use of laser technologies in dental practice and frequent practical application of this approach in the author's clinical work allowed us to distinguish aspects which should be taken into account in the development of a new model of a low-level laser device. Uncontrolled use of laser radiation can have a damaging effect on surrounding tissues. This effect can be prevented by using luminophores in the laser structure - this way the phenomenon of radiation becomes secondary, and conditions in which a physician can easily adjust both the spectrum and the level of energy are formed. These possible device settings, or rather selection of handpieces with different output parameters depending on the combination and composition of luminophores, allow one to use it in various clinical situations. In order to ensure high effectiveness of the new proposed system, the need for maximum cooling has to be taken into account when developing such a system. For this purpose, we suggest using a technology of selective dimensional metal plates' etching, in order to model their typology and the desired hierarchy. By combining such a technology with the methods of accelerated electrolytic metallization, you can minimize the total thickness of a plate up to 200 microns, thus ensuring the most rapid effect of a cooling system with minimal energy consumption. Taking into account the dynamics of modern technological development, as well as constant modifications 
and upgrades of devices used in dental practice in particular, it is logical to develop knockdown laser systems. That is, the possibility of replacing components of a laser device - a fibre optic cable, the system of luminophores, the main working unit of the system - greatly simplifies the task of adapting laser technologies in various clinical situations, and on the other hand, it enables further improvement of a device by using a modified composition of luminophores or a conductive fiber optic cable. In addition, it is this design of the system that is also the most convenient in terms of replacing outdated components, which also allows one to start mass production of each of them. Based on the existing experience, wavelength of the experimental laser system should be equal to $810 \mathrm{~nm}$, given that systems with a wavelength which is close to such a value had the highest clinical effectiveness, judging by the previous studies. That is, by taking into account all the above-mentioned suggestions, it is possible to develop a laser device for dental practice which will have significantly higher effectiveness and mobility, compared with existing systems, while solving the following three important aspects of orthodontic treatment: 1) accelerating the process of orthodontic tooth movement due to the effect on the process of bone remodeling; 2) reducing the duration of dental treatment due to accelerated disposition of teeth in the jaws; 3) reducing the signs of inflammation and pain sensations due to biostimulatory effects on the surrounding oral tissues in the area of direct exposure to radiation.

\section{CONCLUSIONS}

The conducted analysis of the results of published studies on the expediency of using low-level laser radiation in the course of orthodontic tooth movement allowed us to confirm the positive effect of lasers on bone remodeling processes, minimizing the signs of inflammation in the area of teeth that are subject to disposition and reducing the duration of treatment. The suggestions made by the author about the improvement of laser devices can be the basis for developing a system that would be more mobile in terms of construction and more clinically effective, which will expand the use of laser radiation in various clinical situations. In addition, the possibility of replacing and modifying the system of luminophores at the end of a fiber optic cable in the proposed system will contribute to the possibility of constant modification of the device, so that it complies with new scientific and technical developments related to dental practice. In order to determine the effect of laser radiation on orthodontic tooth movement, it is necessary to carry out additional morphometric, X-ray, histological, and clinical studies, which will allow us to distinguish aspects of the effect lasers have on the cells of periodontal ligament space during orthodontic disposition, to analyze the level of changes in architectonics and bone density, the loss of alveolar ridge as a 
result of the effect of mechanical forces, and how accurately this value can be forecast in the conditions of low-level radiation exposure, as well as the effect of lasers on inflammation in the exposed area based on changes in the concentration of cell markers and external clinical manifestations.

\section{REFERENCES}

1. Effects of low-level laser therapy and orthodontic tooth movement on dental pulps in rats / L. B. P. Abi-Ramia, A. Sassa Stuani, A. Sasso Stuani [et al.] // The Angle Orthodontist. - 2010. - Vol. 80. No. 1 - P. 116-122.

2. Effects of low-intensity laser therapy on the orthodontic movement velocity of human teeth: A preliminary study / D. R. Cruz, E. K. Kohara, M. S. Ribeiro [et al.] // Lasers in Surgery and Medicine. - 2004. - Vol. 35. No. 2. - P. 117-120.

3. da Silva Sousa M. V. Influence of low-level laser on the speed of orthodontic movement / M. V. da Silva Sousa, M. A. Scanavini, E. K. Sannomiya [et al.] // Photomedicine and Laser Surgery. - 2011. -Vol. 29. No. 3. - P. 191-196.

4. Doshi-Mehta G. Efficacy of low-intensity laser therapy in reducing treatment time and orthodontic pain: a clinical investigation/ G. Doshi-Mehta, W. A. BhadPatil // American Journal of Orthodontics and Dentofacial Orthopedics. - 2012. Vol. 141, No. 3. - P. 289-297.

5. Low-energy laser stimulates tooth movement velocity via expression of RANK and RANKL / S. Fujiia., M. Yamaguchi, T. Utsunomiya [et al.] // Orthodontics \& Craniofacial Research. - 2008. - Vol. 11. No. 3. - P. 143-155.

G. Ge M. K. Efficacy of low-level laser therapy for accelerating tooth movement during orthodontic treatment; a systematic review and meta-analysis / M. K. Ge. W. L. He, J. Chen [et al] // Lasers in Medical Science. - 2015. - Vol. 30, No. 5. - P. 1609-1618.

7. Effect of low-level laser therapy (LLLT) on orthodontic tooth movement / G. Genc, I. Kocadereli, F.Tasar [et al.] // Lasers in Medical Science. - 2013. - Vol. 28, No. 1. - P. 41-47.

8. Kawasaki K. Effects of low-energy laser irradiation on bone remodeling during experimental tooth movement in rats / K. Kawasaki, N. Shimizu // Lasers in Surgery and Medicine. - 2000. -Vol. 26, No. 3. - P. 282-291.

9. Effects of low-level laser therapy after Corticision on tooth movement arid paradental remodeling / S. J. Kim, S. U. Moon, S. G. Kang [et al.] // Lasers in Surgery and Medicine. - 2009. - Vol. 41. No. 7. - P. 524-533. 
10. Effects of low-level laser therapy on the rate of orthodontic tooth movement / W. Limpanichkul, K. Godfrey, N. Srisuk [et al.] // Orthodontics \& Craniofacial Research. - 2006. - Vol. 9, No.1. - P. 38-43.

11. Marquezan M. Effects of two low-intensity laser therapy protocols on experimental tooth movement / M. Marquezan, A. M. Bolognese, M. T. de Souza Araujo // Photomedicine and Laser Surgery. - 2010. - Vol. 28. No. 6. - P. 757762.

12. Seifi M. Effects of two types of low-level laser wave lengths (850 and $630 \mathrm{~nm}$ ) on the orthodontic tooth movements in rabbit / M. Seifi, H. A. Shafeei, S. Daneshdoost [et al.] // Lasers in Medical Science. - 2007. - Vol. 22. No. 4. - P. 261-264.

12. Yamaguchi M. Low-energy laser irradiation facilitates the velocity of tooth movement and the expressions of matrix metalloproteinase-9, cathepsin K, and alpha, (v) beta (3) integrin in rats / M. Yamaguchi, M. Hayashi, S. Fujita [et al.] // The European Journal of Orthodontics. - 2010. - Vol. 32. No. 2. - P.131-139.

14. Low-energy laser irradiation accelerates the velocity of tooth movement via stimulation of the alveolar bone remodeling / T. Yoshida, M. Yamaguchi, T. Utsunomiya [et al.] // Orthodontics \& Craniofacial Research. - 2009. - Vol. 12, No. 4. - P. 289- 298. 